\title{
Value of neutrophil-to-lymphocyte ratio, monocyte-to-lymphocyte ratio, platelet-to-lymphocyte ratio and red blood cell distribution width in distinguishing between reactive lymphadenopathy and lymphoma in children
}

\author{
Tezol $\mathrm{O}^{1}$, Bozlu $\mathrm{G}^{1}$, Sagcan $\mathrm{F}^{1}$, Tuncel Daloglu $\mathrm{F}^{2}$, Citak $\mathrm{C}^{3}$
}

Mersin University School of Medicine Department of Pediatrics, Mersin, Turkey. ozlemtezol@hotmail.com

\begin{abstract}
OBJECTIVES: The aim of our study was to assess the diagnostic value of neutrophil-to-lymphocyte ratio (NLR), monocyte-to-lymphocyte ratio (MLR), platelet-to-lymphocyte ratio (PLR), and red blood cell distribution width (RDW) in children with lymphadenopathy (LAP).

METHODS: Between January, 2009 and December, 2018, 190 children who underwent excisional lymph node biopsy due to enlarged lymph nodes were included. The clinical and laboratory features of pediatric patients with lymph node enlargement, histopathological examination of the lymph node, and the role of complete blood count parameters in the differentiation of reactive and malignant LAP were analyzed retrospectively.

RESULTS: In total, 139 (73.2 \%) children had pathologically confirmed reactive LAP and 51 (26.8 \%) were diagnosed with lymphoma. Compared with the reactive LAP group, median values for NLR, MLR, PLR, and RDW were significantly higher in children with lymphoma $(p<0.01)$. According to receiver operating characteristic curve analysis performed for distinguishing between reactive LAP and lymphomas, the area under curves of NLR, MLR, PLR, and RDW were $0.75,0.76,0.71$, and 0.61 , respectively.

CONCLUSIONS: Children with histologically proven lymphoma have higher NLR, MLR, PLR, and RDW values than children with reactive LAP. NLR, MLR, PLR, and RDW tests, which can be performed even in primary health care centers, may be useful markers to determine which patients with LAP should be referred to the advanced center at an early stage for biopsy (Tab. 4, Fig. 2, Ref. 26). Text in PDF www.elis.sk. KEY WORDS: Iymphadenopathy, Iymphoma, neutrophil-to-lymphocyte ratio, platelet-to-lymphocyte ratio, monocyte-to-lymphocyte ratio, red blood cell distribution width.
\end{abstract}

\section{Introduction}

Because children's immune systems are constantly stimulated by environmental antigens and microorganisms, their lymph nodes are often palpable. The prevalence of lymphadenopathy (LAP) varies according to age and regional location of lymph node (1, 2). Small occipital and postauricular lymph nodes are common in infants, but not in older children. Cervical and inguinal lymph nodes are more common after 2 years of age than in the first six months of life (2). Epitrochlear and supraclavicular adenopathy are rarely seen at any age. LAP was reported in $44 \%$ of healthy children younger than 5 years and $64 \%$ of children evaluated for

\footnotetext{
${ }^{1}$ Mersin University School of Medicine Department of Pediatrics, Mersin, Turkey, ${ }^{2}$ Mersin University School of Medicine Department of Pathology, Mersin, Turkey, and ${ }^{3}$ Mersin University School of Medicine Division of Pediatric Oncology, Mersin, Turkey

Address for correspondence: Tezol O, MD, Mersin University Hospital Department of Pediatrics, Ciftlikkoy Campus, 33110, Yenisehir, Mersin, Turkey.

Phone: +90.507 .6895011 , Fax: +90.324 .5021518$
}

disease $(2,3)$. LAP can be classified as either localized or generalized depending on whether it occurs in a single region (such as cervical, axillary, or inguinal), or if there are many in more than one non-adjacent region. LAP can occur during the course of various infectious diseases and may develop due to immunological, endocrine, lymphoproliferative, or malignant diseases as well as in consequence of some drug use $(3,4)$.

Unexplained LAPs are a cause for concern to patients and clinicians because of the possibility of malignancy (5). Peripheral LAP in children is usually benign and self-limiting. However, since LAP may also be a manifestation of malignant and autoinflammatory diseases, it is necessary to define the underlying etiology in each patient. The etiology of peripheral lymphadenopathy can often be determined after a full history and physical examination. If the diagnosis remains unclear after clinical evaluation, additional evaluations should be performed $(5,6)$. The urgency and scope of the evaluation is determined by the extent of the patient's appearance and presence of clinical features suggestive of malignancy. Yet, there is no biomarker that can be applied in clinical practice. 


\section{7-292}

In addition to clinical and physical examination findings, the neutrophil-to-lymphocyte ratio (NLR), monocyte-to-lymphocyte ratio (MLR), platelet-to-lymphocyte ratio (PLR), and red blood cell distribution width (RDW) values which can be calculated automatically from a complete blood count have been reported in recent years to be used separately or together in the differential diagnosis and prognosis of inflammatory and malignant diseases (7-9). No study has investigated the role of NLR, MLR, PLR, and RDW in children with LAP. In this study, we evaluated the value of NLR, MLR, PLR, and RDW in distinguishing between reactive LAP and lymphoma in children.

\section{Materials and methods}

In our retrospective study we assessed data from children who underwent excisional lymph node biopsy due to enlarged lymph nodes at the Department of Pediatrics of our tertiary university hospital from January 2009 to December 2018. The approval for the study was obtained from local ethics committee (MEU 2018/314).

The inclusion criteria were: pathologically confirmed reactive LAP or lymphoma after biopsy. The exclusion criteria were: necrotizing granulomatosis, non-necrotizing granulomatosis, other granulomatous diseases, metastatic involvement, autoimmune diseases, lymphoproliferative disorders, or histiocytic disorders. Besides these, the history of antibiotic use within 2 weeks before biopsy was accepted as an exclusion criterion for reactive LAP (Fig. 1).

Clinical and laboratory data were obtained by patient file researching. All of the study children received physical examination before biopsy. Fever history, duration, size, extension of lymph nodes, and biopsy location were determined by reviewing medical records. The size of the lymph node was classified as $<1 \mathrm{~cm}$, $1-3 \mathrm{~cm}$, or $>3 \mathrm{~cm}$. The extension of lymph nodes was classified as localized LAP (a single or multiple but adjacent lymph node involvement) and generalized LAP (more than two non-adjacent
Tab. 1. Demographic and clinical characteristics by study group.

\begin{tabular}{lccc}
\hline Characteristics & $\begin{array}{c}\text { Reactive LAP } \\
(\mathrm{n}=139,73.2 \%)\end{array}$ & $\begin{array}{c}\text { Lymphoma } \\
(\mathrm{n}=51,26.8 \%)\end{array}$ & $\mathrm{p}$ \\
\hline Age, years & $6.75(0.25-18)$ & $14.00(0.5-18)$ & $<\mathbf{0 . 0 0 1}$ \\
\hline Males & $89(64.0)$ & $32(62.7)$ & 0.870 \\
\hline Fever & $37(26.6)$ & $25(49.0)$ & $\mathbf{0 . 0 0 4}$ \\
\hline Duration & & & \\
$\quad$ Acute & $54(38.8)$ & $31(60.8)$ & $\mathbf{0 . 0 2 1}$ \\
Chronic & $85(61.2)$ & $20(39.2)$ & \\
\hline Size & & & \\
$<$ 1cm & $34(24.5)$ & $5(9.8)$ & \\
$1-3$ cm & $68(48.9)$ & $20(39.2)$ & $\mathbf{0 . 0 0 4}$ \\
$>3$ cm & $37(26.6)$ & $26(51.0)$ & \\
\hline Extension & & & \\
Generalized & $18(12.9)$ & $30(58.8)$ & $<\mathbf{0 . 0 0 1}$ \\
Localized & $121(87.1)$ & $21(41.2)$ & \\
\hline Biopsy location, $\%$ & & & \\
Cervical & 61.9 & 56.9 & \\
Submandibular & 17.3 & 7.8 & \\
Abdominal & 9.4 & 11.8 & \\
Axillary & 4.3 & 2.0 & - \\
Supraclavicular & 2.9 & 7.8 & \\
Inguinal & 2.2 & 7.8 & \\
Suboccipital & 2.2 & 0 & \\
Mediasten & 0 & 5.9 & \\
\hline
\end{tabular}

LAP - lymphadenopathy, Data are shown as median (min-max), number (\%) as appropriate

lymph node involvements). The duration of LAP was classified as acute $(\leq 4$ weeks), or chronic $(>4$ weeks).

Complete blood count (CBC) parameters, including leukocyte, neutrophil, lymphocyte, monocyte, and platelet counts (PC) as well as mean platelet volume (MPV), RDW, and hemoglobin values were measured up to 2 days before biopsy. NLR, MLR, and PLR were calculated.

All biopsies were performed by experienced pediatric surgeons; pathologic examinations were performed by experienced

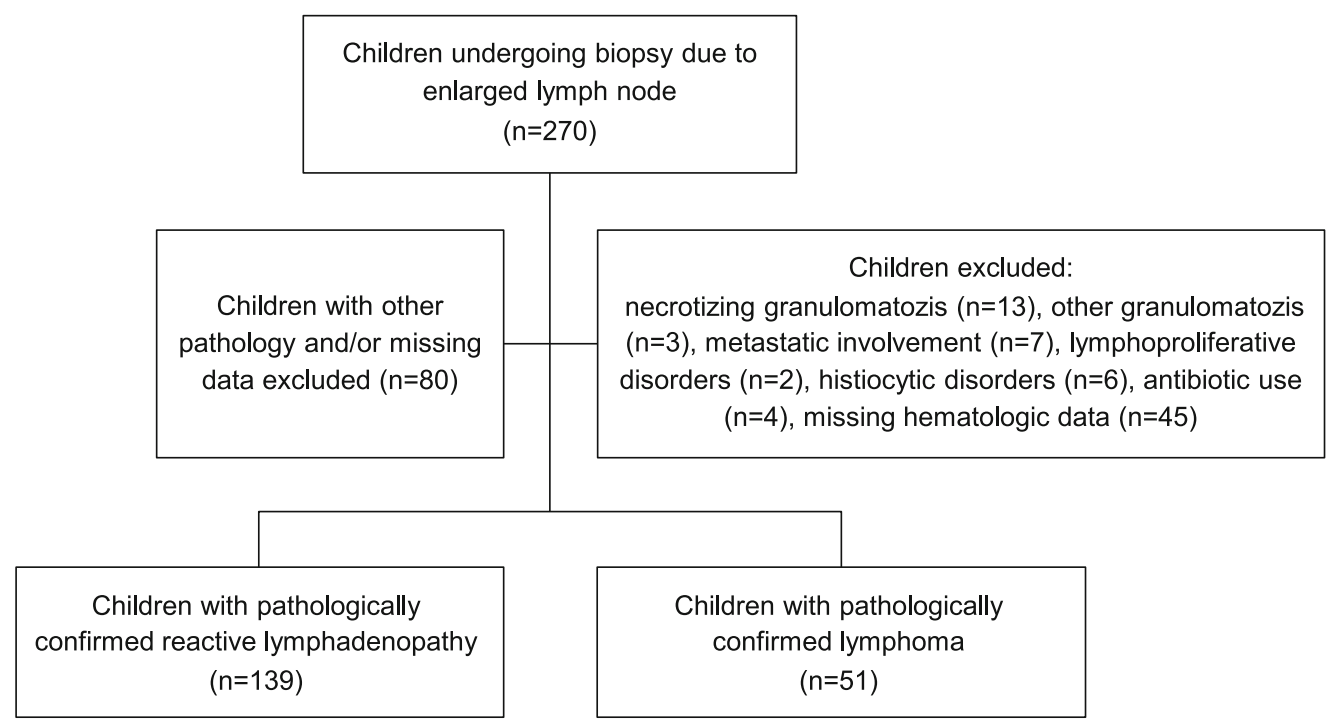

Fig. 1. Selection of the study population. 
Tab. 2. Hematologic biomarkers in reactive LAP and lymphoma groups.

\begin{tabular}{lccc}
\hline Biomarker & $\begin{array}{c}\text { Reactive LAP } \\
(\mathrm{n}=139,73.2 \%)\end{array}$ & $\begin{array}{c}\text { Lymphoma } \\
(\mathrm{n}=51,26.8 \%)\end{array}$ & $\mathrm{p}$ \\
\hline Hemoglobin, g/dL & $12.1(7.7-15.2)$ & $11.1(6.7-13.4)$ & $<\mathbf{0 . 0 0 1}$ \\
\hline RDW, $\%$ & $13.9(11.8-25.1)$ & $14.6(12.2-22.4)$ & $\mathbf{0 . 0 1 7}$ \\
\hline Leucocyte, $10^{3} / \mu \mathrm{L}$ & $8.93(2.83-33.54)$ & $10.31(1.06-28.82)$ & 0.996 \\
\hline Neutrophil, $10^{3} / \mu \mathrm{L}$ & $4.15(0.46-30.61)$ & $6.07(0.50-20.44)$ & $\mathbf{0 . 0 1 9}$ \\
\hline Lymphocyte, $10^{3} / \mu \mathrm{L}$ & $3.18(0.53-14.61)$ & $1.75(0.29-7.99)$ & $<\mathbf{0 . 0 0 1}$ \\
\hline Monocyte, $10^{3} / \mu \mathrm{L}$ & $0.69(0.13-3.26)$ & $0.75(0.10-3.09)$ & 0.412 \\
\hline Platelet, $10^{3} / \mu \mathrm{L}$ & $338(58-1141)$ & $322(40-775)$ & 0.232 \\
\hline MPV, fL & $9.50 \pm 1.13$ & $9.58 \pm 1.14$ & 0.657 \\
\hline MPV/PC ratio & $2.87(0.68-14.66)$ & $2.92(1.17-27)$ & 0.196 \\
\hline NLR & $1.24(0.14-23.55)$ & $4.28(0.17-14.26)$ & $<\mathbf{0 . 0 0 1}$ \\
\hline MLR & $0.20(0.06-1.45)$ & $0.41(0.12-1.63)$ & $<\mathbf{0 . 0 0 1}$ \\
\hline PLR & $105.55(12.94-570.50)$ & $192.94(8.39-775)$ & $<\mathbf{0 . 0 0 1}$ \\
\hline LAP - ly & - &
\end{tabular}

LAP - lymphadenopathy; RDW - red cell distribution width; MPV - mean platelet volume; PC - platelet count; NLR - neutrophil-to-lymphocyte ratio; MLR - monocyte-to-lymphocyte ratio; PLR - platelet-to-lymphocyte ratio Data are shown as median (min-max): mean $\pm \mathrm{SD}$ as appropriate

Tab. 3. Multivariate odd ratios of variables for predicting malignancy in all children.

\begin{tabular}{lccccc}
\hline Variables & $\mathrm{B}$ & Wald & OR & $(95 \% \mathrm{CI})$ & $\mathrm{p}$ \\
\hline Age & 0.214 & 9.398 & 1.239 & $(1.080-1.420)$ & $\mathbf{0 . 0 0 2}$ \\
\hline Duration (acute) & 0.822 & 1.782 & 2.276 & $(0.680-7.613)$ & 0.182 \\
\hline Fever (presence) & 0.851 & 2.007 & 2.341 & $(0.722-7,597)$ & 0.157 \\
\hline Size & & & & & \\
$\quad 1-3 \mathrm{~cm}$ vs 1 cm & -0.462 & 0.267 & 0.630 & $(0.109-3.631)$ & 0.605 \\
$\quad>3 \mathrm{~cm}$ vs 1 cm & 0.385 & 0.213 & 1.470 & $(0.286-7.547)$ & 0.645 \\
\hline Extension (generalized) & 2.679 & 16.027 & 14.576 & $(3.926-54.120)$ & $<\mathbf{0 . 0 0 1}$ \\
\hline Hemoglobin & -0.409 & 4.395 & 0.664 & $(0.453-0.974)$ & $\mathbf{0 . 0 3 6}$ \\
\hline RDW & 0.064 & 0.178 & 1.066 & $(0.792-1.436)$ & 0.673 \\
\hline NLR & -0.034 & 0.086 & 0.966 & $(0.768-1.215)$ & 0.770 \\
\hline MLR & 2.659 & 4.883 & 14.280 & $(1.351-150,965)$ & $\mathbf{0 . 0 2 7}$ \\
\hline PLR & 0.000 & 0.005 & 1.000 & $(0.995-1.006)$ & 0.946 \\
\hline OR & & & & &
\end{tabular}

OR - odds ratio; CI - confidence interval; RDW - red cell distribution width; NLR - neutrophil-to-lymphocyte ratio; MLR - monocyte-to-lymphocyte ratio; PLR - platelet-to-lymphocyte ratio

Tab. 4. Sensitivity and specificity of potential hematologic biomarkers for diagnosis of lymphoma.

\begin{tabular}{lcccccc}
\hline Biomarker & $\begin{array}{c}\text { Cut-off } \\
\text { value }\end{array}$ & $\begin{array}{c}\text { Sensitivity } \\
\%\end{array}$ & $\begin{array}{c}\text { Specificity } \\
\%\end{array}$ & $\begin{array}{c}\text { PPV } \\
\%\end{array}$ & $\begin{array}{c}\text { NPV } \\
\%\end{array}$ & $\begin{array}{c}\text { AUC } \\
(95 \% \text { CI })\end{array}$ \\
\hline RDW & 15.1 & 43.1 & 77.0 & 40.7 & 78.7 & $0.61(0.54-0.68)$ \\
\hline NLR & 3.17 & 60.8 & 85.6 & 60.8 & 85.6 & $0.75(0.68-0.81)$ \\
\hline MLR & 0.29 & 70.6 & 73.4 & 49.3 & 87.2 & $0.76(0.69-0.82)$ \\
\hline PLR & 180 & 56.9 & 87.8 & 63.0 & 84.7 & $0.71(0.64-0.77)$ \\
\hline
\end{tabular}

PPV - positive predictive value; NPV - negative predictive value; AUC - area under the curve; CI - confidence interval; RDW - red cell distribution width; NLR - neutrophil-to-lymphocyte ratio; MLR - monocyte-to-lymphocyte ratio; PLR - platelet-to-lymphocyte ratio

pathologists. According to biopsy results, patients with reactive LAP or lymphomas were grouped as cases with benign or malignant disorder, respectively.

\section{Statistical analysis}

SPSS 21 and MedCalc packages were used for statistical analysis. Continuous variables are statistically described as mean \pm standard deviation or median (min-max) values; categorical variables, are given as numbers (n) and percentages (\%). Shapiro-
Wilk test and histogram were used to test for normality. Independent groups were compared with student t-test, Mann-Whitney U test or Pearson Chi-square test.

Receiver operator curves (ROC) were plotted, and the area under the curve (AUC), 95\% confidence interval (CI), and cut-off values were calculated for NLR, MLR, PLR, and RDW. Sensitivity, specificity, as well as positive and negative predictive values were used to describe predictive properties. The most promising independent variables (univariate analysis) were incorporated into a multivariate analysis as single risk factors, while the binary logistic regression analysis was conducted by calculating the odds ratio to establish the risk for malignancy. The statistical significance level was accepted when $\mathrm{p}<0.05$.

\section{Results}

A total of 190 lymph node biopsy and complete blood count results were included in the study. In total, 139 (73.2 \%) children had pathologically confirmed reactive LAP and 51 (26.8\%) were diagnosed with lymphoma. The median age was significantly different between benign and malignant disorder groups (6.75 (0.25-18.00) and 14.00 (0.5-18.00), respectively; p < $0.001)$. In the malignant disorder group, 31 children (60.8\%) had Hodgkin, and 20 children (39.2\%) had non-Hodgkin lymphoma. The demographic and clinical characteristics of the study children are shown in Table 1.

As compared with the reactive LAP group, the lymphoma group had a significantly higher median value for neutrophil count $(p=0.030)$, and median values for RDW ( $\mathrm{p}=0.017)$, NLR, MLR, and PLR (all $\mathrm{p}<0.001$ ), but a lower median value for lymphocyte count and median value for hemoglobin (both $\mathrm{p}<0.001$ ). No significant differences were found in leukocyte, monocyte, PC, MPV value, and MPV/PC ratio $(p>0.05)$ between the two groups (Tab. 2).

In logistic regression analysis, the overall percent of cases that are correctly predicted by the model was $85.6 \%$. The associations between risk factors and malignancy outcome are shown in Table 3.

The AUCs for RDW, NLR, MLR, PLR, as well as sensitivity, and specificity, and positive and negative predictive values are shown in Table 4. According to pairwise comparisons of ROC curves, the differences between RDW NLR areas and 


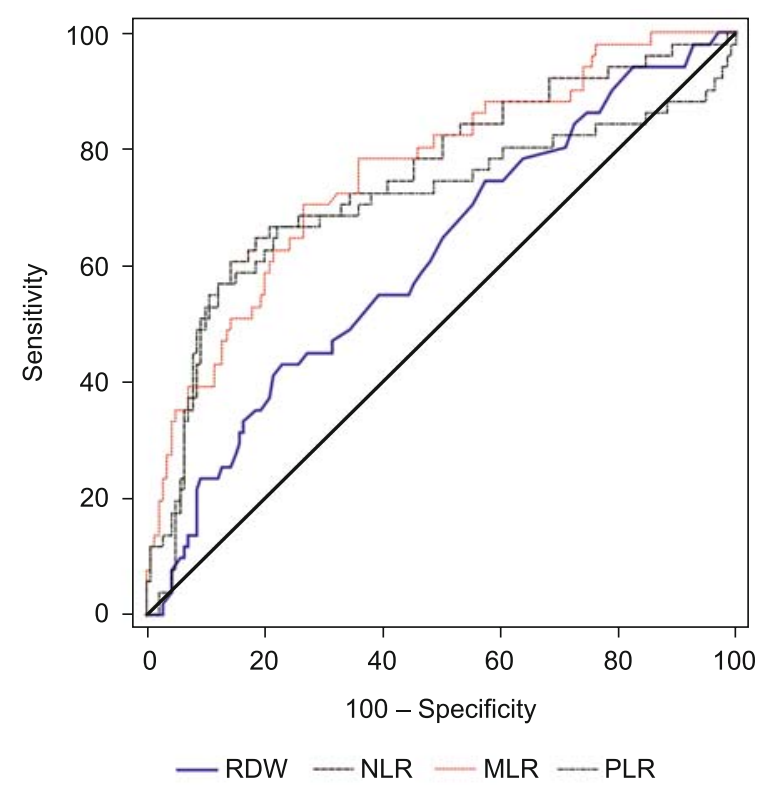

Fig. 2. Receiver operating characteristics (ROC) curves of RDW, NLR, MLR, and PLR in diagnosing lymphoma.

$\mathrm{RDW} \sim \mathrm{MLR}$ areas were statistically significant $(\mathrm{p}=0.031$ and $p=0.017$, respectively); the differences between $R D W \sim P L R$, NLR MLR, NLR PLR, and MLR PLR areas were statistically insignificant $(p=0.147, p=0.744, p=0.206, p=0.183$, respectively). The sensitivity, specificity, and positive and negative predictive values for the combination of RDW, NLR, MLR, and PLR biomarkers were $23.5 \%, 97.1 \%, 75.0 \%$, and $77.6 \%$, respectively. The ROC curves for RDW, NLR, MLR, and PLR are shown in Figure 2.

\section{Discussion}

To our knowledge, this is the first study that has been focused on the role of whole blood parameters in children with LAP. We showed that children in the lymphoma group had significantly higher levels of NLR, MLR, PLR, and RDW than those in the reactive LAP group. On the other hand, we found that there was no difference in the levels of leukocytes, monocytes, PC, MPV, and MPV/PC ratio between the children with lymphoma and reactive LAP.

Peripheral LAP in children may occur during the course of many infectious diseases such as viral, bacterial, fungal, protozoal, and spirochetal $(3,5)$. All patients should have a detailed history and completed systemic examination in order to ascertain facts such as the duration of LAP, whether there was an increase in size, previous history of febrile illness, symptoms of B seen in lymphoma (fever, night sweats, weight loss), animal contact, travel history, vaccinations, medications, and chronic disease history. Further facts and circumstances should be investigated, such as the size of the lymph gland, whether it is mobile or painful, whether there is redness and local heat increase on the skin, localization, and number, as well as additional pathological examination findings such as concomitant organomegaly (10).

The evaluation of the child with LAP should be carried out in steps of approximately four weeks. In cases with localized LAP, the etiology is often determined by history and physical examination. In generalized LAP, some laboratory tests are needed to determine the etiology. Several diagnostic tests have been used in the evaluation of lymph nodes $(2,4)$. The attempt to administer antimicrobial therapy after the initial evaluation has an important role in the diagnosis and treatment. Further investigation should be performed in cases that cannot be explained by antimicrobial therapy and initial tests. Chest X-ray and abdominal ultrasonography, tuberculin skin test, HIV serology, autoantibodies, toxoplasmosis and serological tests for cat scratch disease may be requested $(2,3)$. Biopsy should be requested in cases of LAP the cause of which could not be revealed despite these tests and which persist for more than three weeks. Biopsy can be performed in the early period in patients who have the possibility of malignancy according to physical examination and tests (4). The timing and sequence of tests and the trial of antibiotic therapy vary depending on the localization, distribution, size and accompanying clinical features of the lymph node.

Neutrophils and lymphocytes are essential cellular components of human host defense. The distribution and number of leukocytes depend on the immunological status of the patient and etiology of the infection. Neutrophils are the first line of cellular defense of the innate immune system against infections while lymphocytes play a role in adaptive immunity. The physiological immune response to bacterial infections promotes an increase in neutrophil counts and a decrease in lymphocyte counts (11). Systemic inflammation responses have been associated with cancer development and progression.

Complete blood count, acute phase reactants such as ESR and $\mathrm{CRP}$, and radiological examinations may support or rule out some diagnoses. However, they are not determinative in distinguishing infection from other inflammatory diseases in patients with LAP. Also, they cannot determine the necessity of biopsy for the differentiation of benign and malignant disease $(5,6)$. Therefore, there is a need for a new biomarker that is specific, precise, inexpensive, fast and easy to measure and applicable at every step.

Changes in the distribution of blood cells that develop during the course of infection, inflammation or malignant diseases have led to the idea that blood parameters which are calculated from $\mathrm{CBC}$ may be new biomarkers that can be used in the diagnosis and course of diseases. The role of these biomarkers in different diseases has been reported (7-9). Previous studies on whole blood parameters led us to assess whether NLR, MLR, PLR, and RDW may have a value in distinguishing between reactive LAP and lymphoma in children.

NLR is a marker of general inflammation that can be calculated from complete blood counts. The published studies indicate that increased NLR is valuable in the diagnosis and prognosis of some diseases including cancer and inflammation $(7,8,11)$. NLR has been shown to be a new biomarker of bacterial infections, and increased NLR has been shown to be superior to con- 
ventional infection markers in the early detection of bacteremia (12). In critically ill patients with sepsis and/or trauma, NLR was found to be significantly higher in those with higher risk of mortality determined by clinically applied scores compared to those not at risk $(7,13)$. In our study, the NLR levels were significantly higher in children with lymphoma when compared with the reactive LAP.

In vivo studies have shown that activated platelets can bind to normal endothelium, and secrete potent inflammatory, mitogenic, and chemotactic mediators to promote monocyte migration and inflammatory response. NLR, PLR and MLR are increased by secreted cytokines in systemic inflammation (7). When inflammation is prolonged, the apoptotic cascade is activated in lymphocytes and the lymphocyte count decreases. Based on these changes in inflammation, Chen et al. investigated the diagnostic value of blood parameters as biomarkers in patients with rheumatoid arthritis (RA) and rheumatoid arthritis-associated interstitial lung disease (RAILD) (14). They found that PLR may be applied as a new biomarker for predicting and diagnosing RA and RA-ILD and for distinguishing RA-ILD patients from RA patients and healthy subjects. PLR has also been shown to be useful in monitoring vascular system disorders and malignancies $(15,16)$. High levels of PLT which are shown to serve as a new systemic inflammatory marker, have been shown to be associated with increased mortality risk at the onset of the disease in patients with sepsis (17). We showed that PLT levels in children with lymphoma were significantly higher than in children with the reactive LAP.

In vivo studies showed that monocytes induce tumorigenesis and angiogenesis and inhibit antitumoral immune response. Therefore, it is thought that increased monocyte and decreased lymphocyte counts may be related to tumor processes and so far, the studies investigating the relationship between tumors and MLR support it $(18,19)$. Other studies have shown that MLR is a more useful marker than WBC, neutrophil count and CRP for differentiating patients with bacteremia from those who do not have bacterial infection $(7,20)$. In our study, the mean levels of MLR in children were significantly higher in the lymphoma group than in the reactive LAP group.

RDW is a measure of variation in the size of circulating erythrocytes, which is routinely reported in a standard automated CBC (9). Recently, an extensive research has been done on RDW, and several studies have demonstrated the role of RDW in various forms of inflammatory disease and pathophysiological conditions. RDW has been recently identified as a prognostic factor in various types of cancer (21). It has been found that as for hematologic malignancies, the prognosis in patients with higher RDW is more likely to be poorer than in those with lower RDW (22). We found that RDW levels in children with lymphoma were significantly higher than those in children with the reactive LAP.

We found that $139(73.2 \%)$ children had pathologically confirmed reactive LAP and 51 (26.8\%) were diagnosed with lymphoma. The majority of cases with LAP first apply to the family doctor. The prevalence of malignancy in primary care patients is relatively low $(23,24)$. Biopsy is performed in $2-3 \%$ of patients who apply to pediatrician, and the rate of malignancy in these cases is only $1-2 \%$. The prevalence of malignancy in lymph node biopsies was reported to be $13-27 \%$ in cases referred to advanced centers $(25,26)$.

This study is mainly limited by its retrospective nature. On the other hand, our study has several strengths. This is the first study to examine the values of NLR, MLR, PLR, and RDW in children with lymphoma and reactive LAP.

\section{Conclusions}

Children with histologically proven lymphoma have higher NLR, MLR, PLR, and RDW than children with reactive LAP. When combined with clinical physical examination findings, these markers, which can be calculated automatically from simple blood counts, may be useful for clinicians in referring suspected cases to further centers. This applies especially for the clinicians in centers of primary and secondary levels of care where the serological tests are limited.

\section{References}

1. Jackson MA, Day JC. Lymphatic system and generalized lymphadenopathy. 128. In: Principles and Practice of Pediatric Infectious Diseases. Long SS, Prober CG, Fischer M (Eds). Elsevier, Philadelphia 2018.

2. Twist CJ, Link MP. Assessment of lymphadenopathy in children. Pediatr Clin N Am 2002; 49: 1009-1025.

3. Newman K, Hayes-Jordan AA. Lymph Node Disorders. 844-849. In: Pediatric Surgery. Grosfeld JL, O’Neil JA, Fonkalsrud EW, Coran AG (Eds). Mosby Elsevier, Philadelphia 2006.

4. Rajasekaran K, Krakovitz P. Enlarged Neck Lymph Nodes in Children. Pediatr Clin North Am 2013; 60: 923-936.

5. Weinberg GA, Segel GB, Hall CB. Lymphadenopathy. 637. In: Signs \& Symptoms in Pediatrics, Adam HF, Foy JM (Eds). American Academy of Pediatrics, Elk Grove Village, IL 2015.

6. Ling RE, Capsomidis A, Patel SR. Urgent suspected cancer referrals for childhood lymphadenopathy. Arch Dis Child 2015; 100: 1098-1099.

7. Djordjevic D, Rondovic G, Surbatovic M et al. Neutrophil-to-Lymphocyte Ratio, Monocyte-to-Lymphocyte Ratio, Platelet-to-Lymphocyte Ratio, and Mean Platelet Volume-to-Platelet Count Ratio as Biomarkers in Critically Ill and Injured Patients: Which Ratio to Choose to Predict Outcome and Nature of Bacteremia? Mediators Inflamm 2018: 3758068. doi: 10.1155/2018/3758068.

8. Kumar R, Geuna E, Michalarea V et al. The neutrophil-lymphocyte ratio and its utilisation for the management of cancer patients in early clinical trials. Br J Cancer 2015; 112 (7): 1157-1165.

9. Bozlu G, Taskinlar H, Unal S, Alakaya M, Nayci A, Kuyucu N. Diagnostic value of red blood cell distribution width in pediatric acute appendicitis. Pediatr Int 2016; 58 (3): 202-205.

10. Lanzkowsky P. Lymphadenopathy and Splenomegaly. 463-468. In: Manual of Pediatric Hematology and Oncology. Philip Lanzkowsky (Ed). Elsevier Academic Pres. New York, USA, 2011.

11. Westerdijk K, Simons KS, Zegers M, Wever PC, Pickkers P, de Jager CPC. The value of the neutrophil-lymphocyte count ratio in the diagnosis of sepsis in patients admitted to the Intensive Care Unit: A retrospective cohort study. PLoS One 2019; 14 (2): e0212861. 


\section{7-292}

12. Zahorec R. Neutrophil-to-lymphocyte ratio. Sixteen-year-long history since publication of our article in Bratislava Medical Journal. Bratisl Lek Listy 2017; 118 (6): 321-323.

13. Salciccioli JD, Marshall DC, Pimentel MA et al. The association between the neutrophil-to-lymphocyte ratio and mortality in critical illness: an observational cohort study. Crit Care 2015; 19: 13. doi: 10.1186/ s13054-014-0731-6.

14. Chen Q, Chen DY, Xu XZ, Liu YY, Yin TT, Li D. Platelet/Lymphocyte, Lymphocyte/Monocyte, and Neutrophil/Lymphocyte Ratios as Biomarkers in Patients with Rheumatoid Arthritis and Rheumatoid Arthritis-Associated Interstitial Lung Disease. Med Sci Monit 2019; 25: 6474-6481.

15. Wang Q, Ma J, Jiang Z, Ming L. Prognostic value of neutrophil-tolymphocyte ratio and platelet-to-lymphocyte ratio in acute pulmonary embolism: a systematic review and meta-analysis. Int Angiol 2018; 37 (1): 4-11.

16. Zhao Z, Zhao X, Lu J, Xue J, Liu P, Mao H. Prognostic roles of neutrophil to lymphocyte ratio and platelet to lymphocyte ratio in ovarian cancer: a meta-analysis of retrospective studies. Arch Gynecol Obstet 2018; 297 (4): 849-857.

17. Shen Y, Huang X, Zhang W. Platelet-to-lymphocyte ratio as a prognostic predictor of mortality for sepsis: interaction effect with disease severity-a retrospective study. BMJ Open 2019; 9 (1): e022896.

18. Feng $\mathbf{F}$, Zheng $\mathbf{G}$, Wang $\mathbf{Q}$ et al. Low lymphocyte count and high monocyte count predicts poor prognosis of gastric cancer. BMC Gastroenterol 2018; 18: 148. doi: 10.1186/s12876-018-0877-9.
19. Cao Y, Ni X, Wang Y et al. Clinical and prognostic significance of combined plasma fibrinogen concentrations and the monocyte-to-lymphocyte ratio in patients with ovarian cancer. Ann Transl Med 2019; 7 (11): 242. doi: 10.21037/atm.2019.04.78.

20. Naess A, Nilssen SS, Mo R, Eide GE, Sjursen H. Role of neutrophil to lymphocyte and monocyte to lymphocyte ratios in the diagnosis of bacterial infection in patients with fever. Infection 2017; 45 (3): 299-307.

21. Hu L, Li M, Ding Y et al. Prognostic value of RDW in cancers: a systematic review and meta-analysis. Oncotarget 2017; 8 (9): 16027-16035.

22. Ai L, Mu S, Hu Y. Prognostic role of RDW in hematological malignancies: a systematic review and meta-analysis. Cancer Cell Int 2018; 18 : 61. doi: 10.1186/s12935-018-0558-3.

23. Ferrer R. Lymphadenopathy: differential diagnosis and evaluation. Am Fam Physician 1998; 58 (6): 1313-1320.

24. Lake AM, Oski FA. Peripheral lymphadenopathy in childhood. Ten-year experience with excisional biopsy. Am J Dis Child 1978; 132: 357-359.

25. Soldes OS, Younger JG, Hirschl RB. Predictors of malignancy in childhood peripheral lymphadenopathy. J Pediatr Surg 1999; 34 : $1447-1452$

26. Celenk F, Gulsen S, Baysal E, Aytac I, Kul S, Kanlikama M. Predictive factors for malignancy in patients with persistent cervical lymphadenopathy. Eur Arch Otorhinolaryngol 2016; 273: 251-256.

Received October 28, 2019. Accepted December 2, 2019. 